See discussions, stats, and author profiles for this publication at: https://www.researchgate.net/publication/348707036

\title{
1996-2017 GPS position time series, velocities and quality measures for the CORS Network
}

Article in Journal of Applied Geodesy · January 2021

DOI: 10.1515/jag-2020-0041

CITATION

9 authors, including:

\section{Jarir Saleh}

NOAA/NGS

38 PUBLICATIONS 511 CITATIONS

SEE PROFILE

Kevin Choi

United States Naval Research Laboratory

10 PUBLICATIONS 85 CITATIONS

SEE PROFILE
READS

350

Sungpil Yoon

Amazon

15 PUBLICATIONS 51 CITATIONS

SEE PROFILE

Richard Arthur Snay

National Oceanic and Atmospheric Administration

95 PUBLICATIONS 1,350 CITATIONS

SEE PROFILE

Some of the authors of this publication are also working on these related projects:

Project Gravity: metrology, data processing, tides View project

Project Hodograph View project 
Jarir Saleh*, Sungpil Yoon, Kevin Choi, Lijuan Sun, Richard Snay, Phillip McFarland, Simon Williams, Don Haw, and Francine Coloma

\section{6-2017 GPS position time series, velocities and quality measures for the CORS Network}

https://doi.org/10.1515/jag-2020-0041

Received September 22, 2020; accepted December 31, 2020

Abstract: The CORS network is a volunteer-based network of Global Positioning System reference stations located mainly in the US and its territories. We discuss the most recent comprehensive reprocessing of all GPS data collected via this network since 1996. Daily data for GPS weeks 834 through 1933 were reprocessed leading to epoch 2010.0 coordinates and velocities of 3049 stations aligned to IGS14. The updated realization of the US National Spatial Reference System derived in this work has been in use since late 2019. As a validation of the results, the derived velocity field is compared to several other solutions and to three regional geophysical and geodetic velocity models. These comparisons uncovered unstable stations which move differently than the regional kinematics around them. Once these are ignored, we estimate the horizontal and vertical stability of this updated realization to be better than $\sim 0.3$ and $\sim 0.6 \mathrm{~mm} /$ year, respectively. We use the position residuals and estimated uncertainties from this reprocessing to derive long-term stability measures for all active stations serving longer than 3 years. These measures exposed 60 CORS with the poorest long-term stability, which have been consequently excluded from serving as mapping control.

Keywords: GPS position time series, CORS, velocity, stability, quality measures, mapping

\footnotetext{
*Corresponding author: Jarir Saleh, NGS, Silver Spring, Maryland, USA, e-mail: Jarir.Saleh@noaa.gov

Sungpil Yoon, NGS, Silver Spring, Maryland, USA; and Amazon, Seattle, WA, USA (present address), e-mail: sungpil.yoon@noaa.gov Kevin Choi, NGS, Silver Spring, Maryland, USA; and Naval Research Lab, Washington DC, USA (present address), e-mail: kevin.choi@noaa.gov

Lijuan Sun, Phillip McFarland, Don Haw, NGS, Silver Spring, Maryland, USA, e-mails: lijuan.sun@noaa.gov, phillip.mcfarland@noaa.gov, don.haw@noaa.gov

Richard Snay, NGS, Silver Spring, Maryland, USA (Retired), e-mail: rssnay@aol.com

Simon Williams, National Oceanography Centre, Liverpool, UK, e-mail: sdwil@noc.ac.uk

Francine Coloma, NGS, Boulder, Colorado, USA, e-mail:

Francine.Coloma@noaa.gov
}

\section{Introduction}

The volunteer-based, multi-purpose NOAA CORS Network, currently consisting of $\sim 1900$ operational (and $\sim 500$ decommissioned) GPS stations (see dynamic map at https:// geodesy.noaa.gov/CORS_Map/), was established by a cooperative effort involving 235 organizations from various US government agencies, academia, and private sector. This network was designed to support a broad spectrum of GPS science and engineering applications, all of which require highly accurate coordinates at all times [1]. Although heterogeneous in the sense that its stations are built and operated by many organizations, this network is supposed to meet high quality and stability specifications. NGS uses the CORS network for defining and accessing the US NSRS, the infrastructure for all surveying and mapping activities in the country [2]. It is necessary, therefore, to ensure that the reference-coordinates and velocities of all CORS are as accurate and their uncertainties as realistic as possible. The quality/stability of the different stations of this heterogeneous network has never been quantified.

NGS intermittently reprocesses all collected daily GPS data via this network in a large campaign called "MultiYear CORS Solution" or MYCS. The first reprocessing, MYCS1, was released in 2011 [3]. We discuss the second reprocessing, MYCS2, completed in 2018. We first describe the data and their quality control procedures in section 2 . The creation of the daily position time series by a dualstep adjustment is described in section 3. The combination of the solutions, velocity computation, and the alignment to IGS14 [4] is described in section 4. This includes some details of discontinuity detection, the handling of nonlinear motion, velocity constraints, and the computation of reference-position and velocity uncertainties taking timecorrelated noise and surface loading effects into account. The examination and validation of the accuracy of the derived velocity field is described in section 5 . This validation uncovered spatially unstable stations where significant disagreements with regional deformation patterns occur, likely due to localized crustal motion. In section 6, measures of long-term CORS quality are introduced based on the long-term stability of each station over the years. These measures are used for ranking all stations in terms 
of their fitness to serve as geodetic control for mapping. A summary ends the presentation in section 7 .

Supplementary material is separated from the main manuscript to keep it concise, but hoping that it could help in explaining and clarifying some aspects of our work and presentation. The supplementary material includes five sections, eighteen figures and three tables. Section S1 describes the automated edge detection filter we used. Section S2 describes the comparison of MYCS2 velocities to other solutions. Section S3 describes the fuzzy clustering method we used to separate spatially stable from unstable CORS. Section 44 presents some examples of long-term unstable CORS, inadequate for mapping control. Finally, section S5 describes differential entropy as a complexity, i.e. instability, measure of position residual time series, which is hopefully more sensitive than the RMS to all stochastic properties of the time series, including time-correlated noise.

\section{CORS data}

Acceptance of a station into the CORS network requires that it must meet the NGS CORS quality guidelines $[5,6]$, which recommend stability and monumentation requirements. A CORS is equipped with a dual-frequency receiver and its antenna must have absolute calibration information from the IGS. When a partner agency requests that its station becomes a CORS, they provide documents including photos of the equipment and settings, a metadata log file and a link to download data. NGS examines the photos for sky obstructions, ensures that the serial numbers in the photos match those in the log file, ensures that the settings are stable and conform to the guidelines and that antennas are properly mounted and away from interference. Three days of data are downloaded and run through program TEQC [7] to ensure that at least $90 \%$ of all possible data have been logged and multipath values MP1 and MP2 are not excessive. A CORS should track data down to at least 5 degrees above the horizon, the cycle slips must not be excessive, no cycle slips above elevation angle of 40 degrees should occur, and the proposed station should be $\sim 70 \mathrm{~km}$ away from currently existing CORS.

In MYCS2, daily RINEX data are passed through TEQC again. If the number of observations in a daily RINEX file, the number of outliers, the percentage of data in the file and the number of cycle slips fall outside certain thresholds then the station is rejected from the daily analysis. Otherwise, the RINEX file's header records are

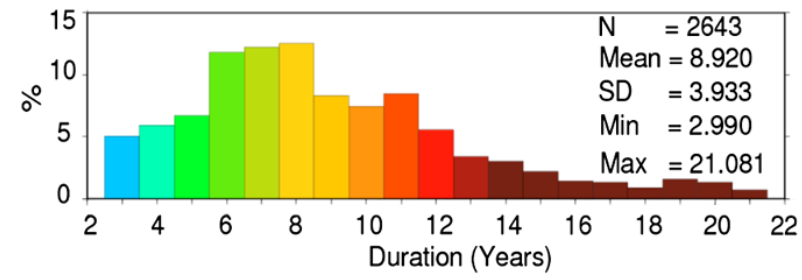

Figure 1: Histogram of time series length in years (bins are centered on the number of years) for MYCS2 stations serving $>3$ years. The average life span of the CORS network is $\sim 9$ years. $82 \%$ of stations served for 5 years or more.

modified replacing the antenna type, geocentric Cartesian coordinates and eccentricity values with those extracted from our database, and the file is decimated to 30 seconds. The RINEX files are then passed through the legacy program "clockprep" to remove millisecond clock jumps, mostly in older data (https://gps.alaska.edu/jeff/ software.html), and "cc2noncc" to correct C1 code biases (https://www.nrl.navy.mil/ssdd/sites/www.nrl.navy.mil. ssdd/files/files/cc2noncc.f). All L1 and L2 phase and code observations are used in our processing down to elevation angle of $10^{\circ}$. The code observations are used for receiver clock synchronization and to help in ambiguity resolution with the Melbourne-Wübbena (MW) combination [8]. Figure 1 shows the lifetime histogram (bins centered on the year) for all MYCS2 stations serving more than 3 years and statistics in years of their time series length. The average lifetime in the CORS network is $\sim 9$ years.

\section{CORS daily coordinate adjustments}

To avoid biases due to poorly understood and hence neglected regional correlations, the daily adjustment procedure consists of two separate adjustments: the orbits/global adjustment and the CORS adjustment. The former is similar to the procedure that results in the final GPS orbits which the NGS analysis center submits weekly to the IGS. It is a global adjustment computed for establishing a fully consistent Earth Centered Earth Fixed (ECEF) reference frame to be used for the CORS adjustment [3]. The CORS adjustment connects the CORS network resulting in positions relative to that global frame. Both adjustments are computed using the NGS software PAGES [9]. The ambiguity resolution in MYCS2 relies on the ion-free and Melbourne-Wübbena combinations instead of the ionfree and wide lane combinations used in previous processing. This has improved the NGS contribution to the IGS 
since Week 1820, removing biases between NGS products and those of other IGS analysis centers (Rebischung 2015, personal communication). Processing daily GPS data from 3049 stations collected over 21 years requires large computing power. NGS utilized the MS Azure $@$ cloud computing service. Using as many as 50 virtual servers simultaneously, the entire daily position adjustments data processing took about 3 weeks.

The global adjustment employs a global network of IGS stations with a fairly homogeneous geographic distribution. The modeling of force fields affecting the dynamics of GPS satellites and stations adheres to IERS conventions [10]. The second order ionospheric effect has not been modeled at NGS yet. Surface loading due to atmospheric, hydrological and non-tidal ocean mass are not modeled at NGS as usual for most IGS analysis centers. Estimated parameters include the satellites' geocentric positions and velocities, five solar radiation pressure scaling parameters in three orthogonal directions, hourly tropospheric wet zenith path delay corrections, geocentric station positions, and earth orientation parameters (EOPs). To define an ECEF frame, the positions of all stable IGS core stations are constrained in a no net rotation (NNR) inner-constraints manner. Stable IGS core stations are those which are neither experiencing postseismic motion nor have jumps in their time series between the time of the release of the IGS14 reference frame and the day of the reprocessed data. The NNR constraint defines the orientation of the global frame thereby completing its definition. The baselines processed in the global adjustment are determined by a Delaunay triangulation of the global network [3]. Figure S1 of the supplementary material presents an example of an IGS network used in the global adjustment and the Delaunay triangulation that defines the processed baselines. The processing is done in individual regions separately, where a region consists of a star-like portion of the Delaunay triangulation, including a hub and the baselines connected to it. The processing of regions is done in parallel, resulting in all regional normal matrices, which are then combined, the NNR constraint is applied and the system is inverted. If necessary, the process is iterated until convergence.

The CORS daily adjustment is computed next. To tie the CORS network to the aforementioned global frame, daily GPS data from all IGS stations of the global adjustment are processed together with the CORS data, forming a global network that is dense ( 2400 stations) in the US (Figs. S1 and S2). Figure S2 of the supplementary material schematically describes the connection of CORS to IGS stations and the CORS-baselines processed in a CORS daily adjustment. The data processing of this network broadly follows that of the global adjustment with two exceptions.
The CORS baselines are defined by finding the closest IGS station and forming a baseline between the two, and the datum is defined by constraining all global parameters (orbits, EOPs, daily IGS station coordinates and hourly tropospheric zenith path delay parameters at those stations) to values which resulted from the global adjustment. This introduces the same global reference frame into the CORS adjustment, resulting in daily positions of all CORS relative to the global NNR frame in the form of daily SINEX files, including all daily spatial correlations between all coordinates.

The quality of the MYCS2-derived orbits and IGS station coordinates is verified by comparing them to IGS products. Finally, weekly SINEX files are computed as weighted means of the daily SINEX files, iteratively inspecting daily coordinates as compared to the weekly mean and using thresholding to remove daily outliers. This procedure resulted in 1100 weekly SINEX files for weeks 834 to 1933 relative to global, weekly NNR reference frames.

\section{Combination of the weekly SINEX files, velocity estimation and alignment to IGS14}

The combination of the weekly SINEX files and their alignment to IGS14 is computed using IGN's software CATREF [11]. We analyze weekly rather than daily position time series because, for mapping, there is no practical benefits to be gained, only computational burdens, from analyzing daily position time series. Basically, combining a time series of weekly SINEX files and aligning them to IGS14 consists of two steps. First, transforming each weekly SINEX file into IGS14 which results in spatially correlated position time series of all stations and second, parameterizing the trajectory of each station as piecewise linear, with a reference-epoch position and linear velocity for each piece. Such parameterization can be used to predict future positions relative to IGS14. Because of the spatial correlations between all position time series (about a quarter of all correlations are larger than 0.25 and correlations of 0.75 are not uncommon), both of the above steps are done simultaneously in one large adjustment procedure.

We detected discontinuities and outliers iteratively. The first iteration starts without declaring any discontinuities or outliers, and results in "raw" position residuals (e. g., Fig. S3) which uncover most sizable discontinuities and outliers. An automatic edge detection algorithm called "the weak elastic string" $[12,13]$ is then applied to 
the "raw" position residuals of that iteration to detect discontinuities (see section S1 and Fig. S3). These are modeled in the following iteration, edge detection is applied to its position residuals to detect smaller discontinuities, and the iterations go on until no more discontinuities are found. The weak elastic string misses discontinuities in difficult situations when the edge is immersed in noise or when consecutive edges occur due to the presence of large slopes in the residuals. Therefore, in the last few iterations, visible discontinuities and outliers are detected manually $[14,15]$. Figure S4A shows the position residual time series of station AB14 in Alaska in an initial iteration and Fig. S4B shows the final residuals after all spikes due to snow accumulation on the antenna had been deleted. Before the last few iterations, we replace our discontinuities for all IGS14 stations by the IGS-detected discontinuities. For stations such as ALRT (located in northeastern Canada, close to Greenland), where our detected discontinuities conflicted with those of the IGS [4], the station was not treated as an IGS14 (reference) station. Because of all discontinuities occurring at many stations, MYCS2 has 8221 solutions even though it involves only 3049 stations. Our discontinuity detection procedure did not rely on information about equipment changes until the very last step because many equipment changes do not cause discontinuities in position time series. Once all discontinuities are detected, their dates are correlated with the dates of equipment changes and, unless a clear discrepancy is found, the detected discontinuity dates are replaced by the logged equipment change dates. Along the west coast, in Alaska and in a few other seismically active spots around the world, detected discontinuity dates are also correlated with dates of large earthquakes and when they match, the detected date is replaced by the recorded date of the earthquake. Thus, detected discontinuity dates were often corrected by several days and sometimes by a week or even two.

For the small minority of stations with nonlinear motion, we linearize their motion by inserting discontinuities which divide their position time series to short linear segments, each preferably longer than three years. To account for the nonlinearity, the velocity of each segment is free to adjust as dictated by the data. The boundaries of the linear segments are visually and iteratively determined to try to minimize the curvature of the position residuals while keeping a reasonable segment length, and deleting data when segments are too short. An example of the performance of this linearization strategy is shown in Fig. S5, where the black curves represent the predicted IGS14 position of station AREQ, reconstituted using the IGS14 reference-epoch position, velocity and postseismic deformation model $[4,16]$. The blue line in Fig. S5 represents a reconstitution using our linearized solution. AREQ, being located near the Nazca subduction zone in southern Peru, is one of the worst cases of the performance of the linearization strategy. The RMS difference between the linearized (blue curve) and nonlinear trajectory (black curve) of Fig. S5 is $1.8 \mathrm{~mm}$ in the East, $1.3 \mathrm{~mm}$ in the North and $2.5 \mathrm{~mm}$ in Up component, and the maximum differences are 6.2, 4.7 and $5.7 \mathrm{~mm}$, respectively. The nonlinear motion of this small minority of stations is relevant to our analysis for one single purpose alone: avoiding their degrading effect on the NSRS. We exclude these stations from defining the NNR frames in the daily adjustments, we remove them from the IGS14 SINEX file before using it as a datum for the alignment, we include these stations in all our DoNot-Use files as geodetic control for our online positioning engines, and we advise the mapping community to avoid them as geodetic control in all mapping activities.

Velocity constraints are applied to force velocities of the solutions on both sides of every manmade discontinuity to be equal. Discontinuities due to unknown causes ( $15 \%$ of all discontinuities) are considered manmade while discontinuities caused by natural disturbances (e. g., earthquakes, postseismic motion, nonlinearities due to manmade non-uniform subsidence and other nonlinearities) are allowed to change the velocity. In the final analysis, 2836 out of 3049 MYCS2 stations have a single velocity. In addition, during the 21 years of CORS history analyzed in the MYCS2, 389 pairs of stations have occupied the same physical point or points close to one another ( $<50$ meters apart), usually following and continuing each other in time. Assuming that localized CORS motion relative to the bedrock is negligible, we constrain the velocities of such pairs to be equal.

To minimize aliasing due to periodic surface loading effects $[17,18,19]$ in the combination and alignment adjustments, we split the procedure to three steps. First, we estimate Helmert's transformation parameters necessary for aligning the 1100 weekly SINEX files to IGS14, adopting the subnet method [20]. The transformation parameters are thus computed avoiding regional correlations (or so-called common-mode errors) by relying on a homogeneously distributed global sub-network of stable core IGS stations (Fig. S6) as a representation of IGS14, and the scale factors were not estimated to minimize the magnitude of possible aliasing, especially in the heights. In the second step, the combination is computed constraining the transformation parameters to their values obtained by the subnet method. A final, more accurate, alignment to IGS14 is finally computed by aligning the combined SINEX file of the second step to IGS14 using all stable IGS stations, 


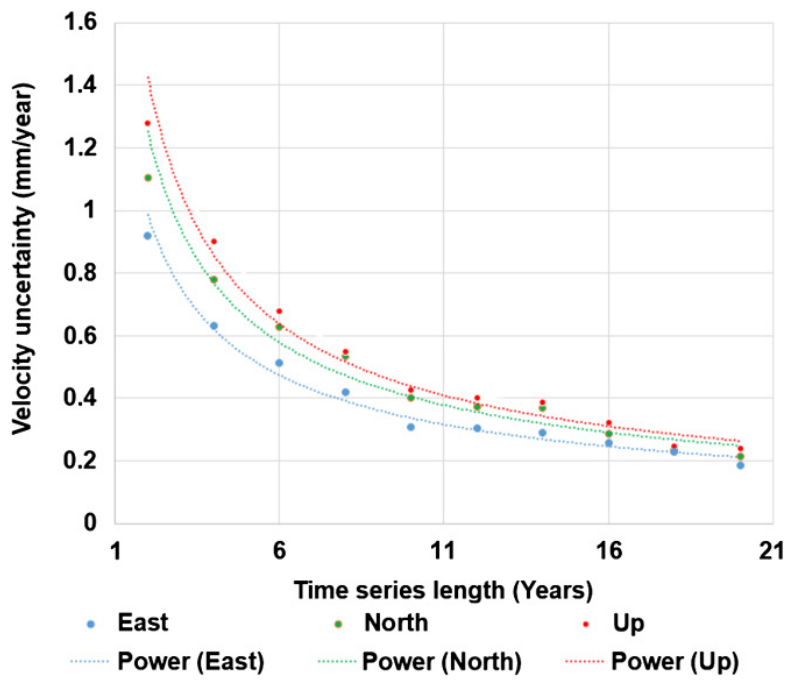

Figure 2: Velocity uncertainty, E (blue), N (green) and U (red) as function of time series length, based on 2-years bins $(1-3,3-5, \ldots, 19-21$ years). The discrete RMS dots refer to the middle of each bin. The curves are least-squares fits (by Excel) to the dots using power functions.

rather than only the subnet. Also, in the final analysis, we discard estimated velocities of stations serving less than 3 years [21] and replace them by predicted velocities using the TRANS4D velocity model [22, 23].

To prevent unmodeled periodic loading effects from inflating error estimates and to account for time correlations in the time series $[24,25,26,27,28,19,29]$, we use the CATREF output to reconstruct adjusted geocentric Cartesian position time series for all stations. These time series are free of outliers, linearized, have well known discontinuity dates, and contain surface loading effects and time-correlated noise. Program CATS [30] is then used to analyze these series, modeling and removing discontinuities, approximating loading effects by an annual and semiannual sinusoids and removing them, and separating white from colored noise assuming that all noise is zero-mean Gaussian and assuming that the colored noise can be described by a general power-law model [29]. Figure 2 shows the orders of magnitude of the CATS-derived CORS velocity uncertainties and their decay with increasing lifespan. These uncertainties are slightly worse than those of IGS stations [29], reflecting the differences in data quality and stability between the CORS and the IGS networks.

Fig. 3 presents a histogram of derived spectral indices of the colored noise, showing a significant spread around flicker noise (index $=-1.0$ ). There are 23 stations, excluded from Fig. 3, which have X, Y or Z spectral indices smaller than -3.0. Every one of these sites has weaknesses that lead to its extreme spectral behavior, too short discontinuous lifespan is chief among them, and most of these stations are decommissioned. Among the rest of the stations (Fig. 3), there are 58 that show random walk or smaller in$\operatorname{dex}(-3.0 \leq$ index $\leq-2.0)$. Most of these stations have abnormalities of some kind. Of these, 29 have timespan shorter than a total of 150 weeks, usually spread over several years with significant data gaps, and the great majority are decommissioned. Also, many of these stations have variable seasonal behavior, improperly sampled by the data due to short life span and data gaps. We also found one missed outlier in two stations, a missed jump of $\sim 10 \mathrm{~mm}$ before the last few months of the life of station EIL2, and unmodeled nonlinearity in the Up components of stations MOSV and NEYK at the beginning of 2013. Of the 58 stations, those with the longest lifespan have either experienced large earthquakes and postseismic motion (e. g., VANU, NTUS, ASPA, TONG, SAMO and AREQ), manmade non-uniform subsidence (e. g., BKR2, P105 and P565) or seriously unstable behavior such as MNEY likely due to failing hardware, NAUS due to massive hydrological seasonality, and TXTR probably due to unstable monumentation causing large horizontal drifts. Finally, of all stations represented in Fig. 3, there are 395 stations with spectral indices between -0.5 and 0.0. Unlike the previous case, the residual time series of the longest serving ( $>7$ years) 100 of these stations have no abnormalities. In fact, the great majority of their residual time series are less noisy and less seasonal than average, with no significant nonlinearity (e.g., STJO, PDEL, CAGS, LAMT, UVFM, GUST and PIMO). Of the 395, there are 110 stations with shorter life spans than 3 years, which happen to have the largest spectral indices, closest to 0.0. These also happen to have corresponding white noise amplitude close to zero because the maximum likelihood estimation method used in program CATS has difficulties separating the colored from the white noise in short time series. We have not used extreme spectral indices between iterations as a tool for detecting abnormalities, such as missed outliers, edges, excessive seasonality or mismodeled nonlinearities, but such tool would probably be useful in future work.

\section{The estimated velocity field and detecting unstable CORS}

We first compare our estimated velocity field to those of several other velocity solutions. These comparisons are summarized in section S2 and Fig. S7 of the supplementary 




Figure 3: Histograms of spectral indices of the general power-law noise in $X, Y$ and $Z$. The values refer to the geocentric Cartesian components since the input to program CATS are Cartesian positions. Extreme negative values are due to short duration, long data gaps, and instability.

material, and show no outstanding differences between the different solutions.

To examine the fine structure of the MYCS2 estimated horizontal velocity field, we compute velocity residuals (Fig. 4) with respect to the NA plate Euler pole located at latitude $2.3^{\circ} \mathrm{S}$ and longitude $86.0^{\circ} \mathrm{W}$ with an angular velocity of $0.2010^{\circ}$ per million years [31]. This Euler pole is independent of any NA intraplate deformations, including horizontal GIA, and any related assumptions [31]. Figure 4A shows residual velocities to the east of the Rocky Mountains (longitude $100^{\circ} \mathrm{W}$ ), computed based on 914 CORS and excluding 100 stations that exhibit localized motion uncharacteristic of the surrounding stations (see section S3 and Fig. S9). These velocity residuals exhibit clear trends but still contain a significant amount of noise. To filter out this noise, we grid the velocity residuals of Fig. 4A on a $1^{\circ} \times 1^{\circ}$ grid using a weighted mean of the 200 nearest neighbors, 50 in each quadrant, where weights are taken as the inverse square distance of the neighbor from the gridded corner, and where the East and North components are gridded separately. The smoothed velocity residuals produced by this simple procedure (Fig. 4B) are almost identical to the intraplate velocity field of [31], and are attributed entirely to horizontal GIA. The agreement between our intraplate deformations (Fig. 4B) and those derived in [31] is a validation of the accuracy of our estimated velocity field to the east of the Rocky Mountains. In addition, these velocity residuals serve two more important purposes. First, we used them to expose the $\sim 100$ stations excluded from Fig. 4 and shown in Fig. S9A, which we deem as the "spatially unstable CORS" east of the Rock- ies. This is because the motion of each of these stations differs significantly from the regional crustal motion around it. We detected and isolated these stations by an iterative process of the previously mentioned noise smoothing, followed by subtracting the smoothed residuals from the original velocity residuals resulting in vectors of "velocity noise", and then clustering [32] these velocity noise vectors into two clusters, the spatially stable and the spatially unstable. As usual for most automations, this procedure was completed by a visual intervention to clean up a few questionable clustering decisions. Section S3 of the supplementary material describes the fuzzy clustering algorithm used for this purpose. The second reason for the importance of the velocity residuals is that they lead to a reliable estimate of the noise level of the MYCS2 velocity field, which can be computed by subtracting the smoothed velocity residuals of Fig. 4B, consisting purely of horizontal GIA, from the original velocity residuals of Fig. 4A. For both horizontal components, the means and medians of this noise are $\sim 0.0 \mathrm{~mm} /$ year, the maximum value is $\sim 1.0 \mathrm{~mm} /$ year, the (equal) means and medians of the absolute values are 0.18 for the East and $0.20 \mathrm{~mm} /$ year for the North, and the RMS values are 0.25 and $0.28 \mathrm{~mm} /$ year for the East and North components respectively.

To the west of the Rocky Mountains and in Alaska, we compute horizontal velocity residuals with respect to two velocity models: TRANS4D v0.2 [22, 23] and GSRM v2.1 [33]. The TRANS4D model in western CONUS is based on block-fault models constrained to smoothly match the velocities of continuous GPS stations and other trusted data. In Alaska and western Canada, the TRANS4D model is comprised of separate models to address tectonic, postseismic and GIA motions [22]. Figure 5A shows our horizontal velocity residuals relative to the TRANS4D velocity model (NGS-TRANS4D) at $~ 350$ active CORS serving more than 3 years, excluding $\sim 25$ such stations because they exhibit localized behavior uncharacteristic of their neighbors' (Fig. S9B).

The GSRM v2.1 model [33] is a geodetic plate motion model which estimates (or in some cases, considers and constraints) the angular velocity vectors of 50 tectonic plates while allowing plate boundaries ( $14 \%$ of the Earth's surface) to deform. We used a $0.1^{\circ} \times 0.1^{\circ}$ velocity grid, disseminated with the supporting information of GSRM v.2.1, to predict velocities at the locations of the previously mentioned $\sim 350$ CORS, and the resulting velocity residuals are shown in Fig. 5B. Notice the similarities of the plots and statistics of the two residual fields of Fig. 5, indicating that the combination of velocity errors and inaccuracy of modern horizontal velocity modeling in California and western Oregon and Washington are 

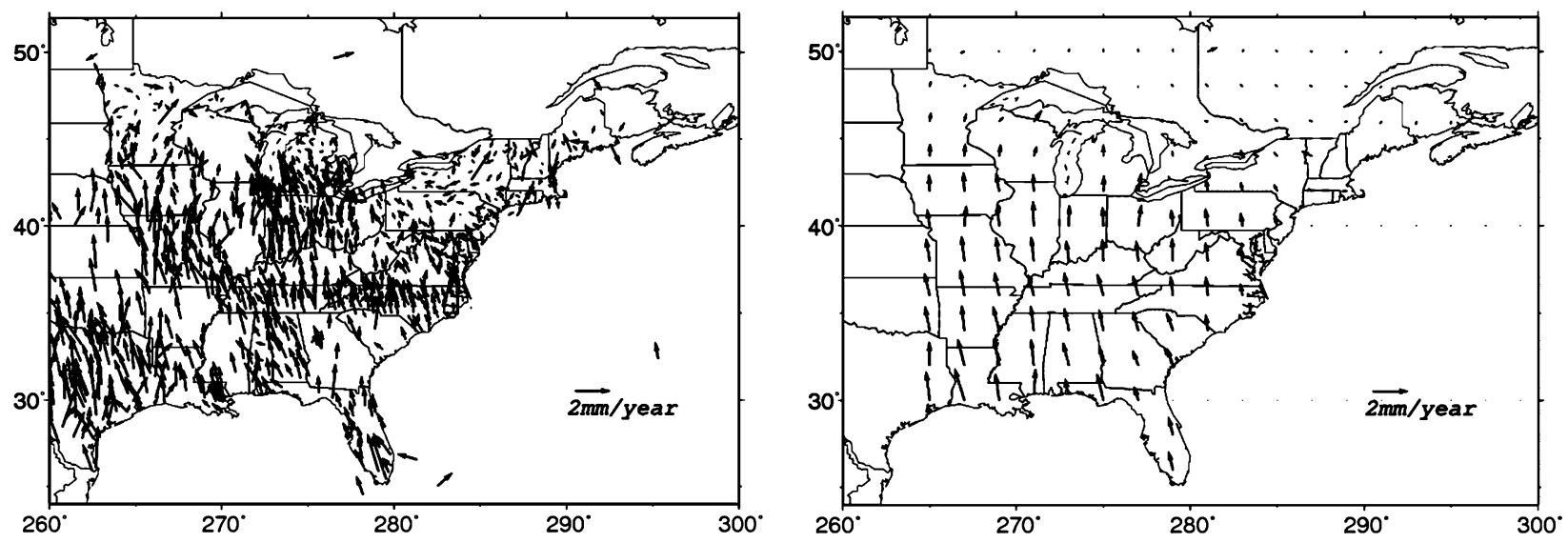

Figure 4: (A: left) Residual velocity, east of longitude $100^{\circ} \mathrm{W}$, with respect to the NA plate Euler pole located at latitude $2.3^{\circ} \mathrm{S}$ and longitude $86.0^{\circ} \mathrm{W}$ and an angular velocity of $0.2010^{\circ}$ per million years [31]; (B: right) A smoothed version of the above velocity residuals on a $2^{\circ} \times 2^{\circ}$ degree grid.

$\sim 0.5-0.7 \mathrm{~mm} /$ year. The velocity residuals in Alaska (Fig. 6) are larger with RMS of $\sim 1.4-2.2 \mathrm{~mm} /$ year. Dissimilarities between the $\sim 0.5 \mathrm{~mm} /$ year level residuals of both models in Fig. 5 and the $\sim 1.5 \mathrm{~mm} /$ year residuals of Fig. 6 are beyond this paper, which presents a mapping datum. Table S2 lists all the spatially unstable stations shown in Fig. S9. The horizontal motion of these sites differs from motion patterns around them either in magnitude or orientation. Although we detect these sites based on their horizontal velocity alone, it turned out that for many of them (Fig. S9A), the horizontal instability is only a consequence of the non-uniform vertical motion, e.g., stations located in subsidence zones in California's Central Valley, southern Texas and Louisiana and along the eastern coastline of the US and the shorelines of the Great Lakes.

The stability, i.e. predictability of future positions, is very important for mapping. Based on the velocity comparisons mentioned above, we can make the statement that the stability of the MYCS2 realization is better than $\sim 0.3 \mathrm{~mm} /$ year in each of the horizontal components, which is consistent with [34]. In California and western Oregon and Washington, the combination of horizontal velocity uncertainty and inaccuracy of modern horizontal deformation modeling (at least by both the TRANS4D v0.2 and GSRM v2.1 models) is $~ 0.5-0.7 \mathrm{~mm} /$ year and in Alaska it is $\sim 1.4-2.2 \mathrm{~mm} /$ year. For a frame that is realized every $\sim 10$ years, these horizontal stability estimates are more than sufficient for mapping and monitoring horizontal crustal motion faster than $\sim 1.0 \mathrm{~mm} /$ year. We cannot make a similar statement about vertical stability based on kinematics. However, we comment in the next section on an estimate of vertical stability.

\section{CORS long-term stability based on position time series}

We know that relying on the complexity of position time series and kinematics can only expose the poorest stations. Nevertheless, the quality of the CORS network is not homogeneous and has never been evaluated before. Furthermore, we show in this section and in the supplementary material that relying on position time series alone uncovers $\sim 60$ unstable CORS, some of which due to malfunctioning equipment and unstable monumentation, which should have never been used as geodetic control for mapping.

We base the long-term stability of a station on its (zero-mean) position residual time series and the uncertainties of its estimated reference-epoch position and velocity. The complexity of the position residuals reflects the level of random noise, systematic errors, previously missed small outliers and jumps and residual nonlinearity modeling such as residual postseismic behavior (e.g., Fig. S5). The estimated uncertainties of the different solutions of a station reflect the price paid in confronting the station's volatility, e. g., crustal and environmental instability and nonlinearity, or the so-called "distribution of discontinuities". To capture the entire complexity of both residuals and uncertainties, we define a "modified position residuals" time series as:

$$
c_{t}=\rho_{t}+P_{0}+v *\left(t-t_{0}\right)
$$

where $t$ is an arbitrary epoch, $t_{0}=2010.0, \rho_{t}$ is the CATREF residuals, $P_{0}$ and $v$ are zero-mean random quantities with stochastic properties identical to those of the referenceepoch position and velocity of the corresponding solution. 

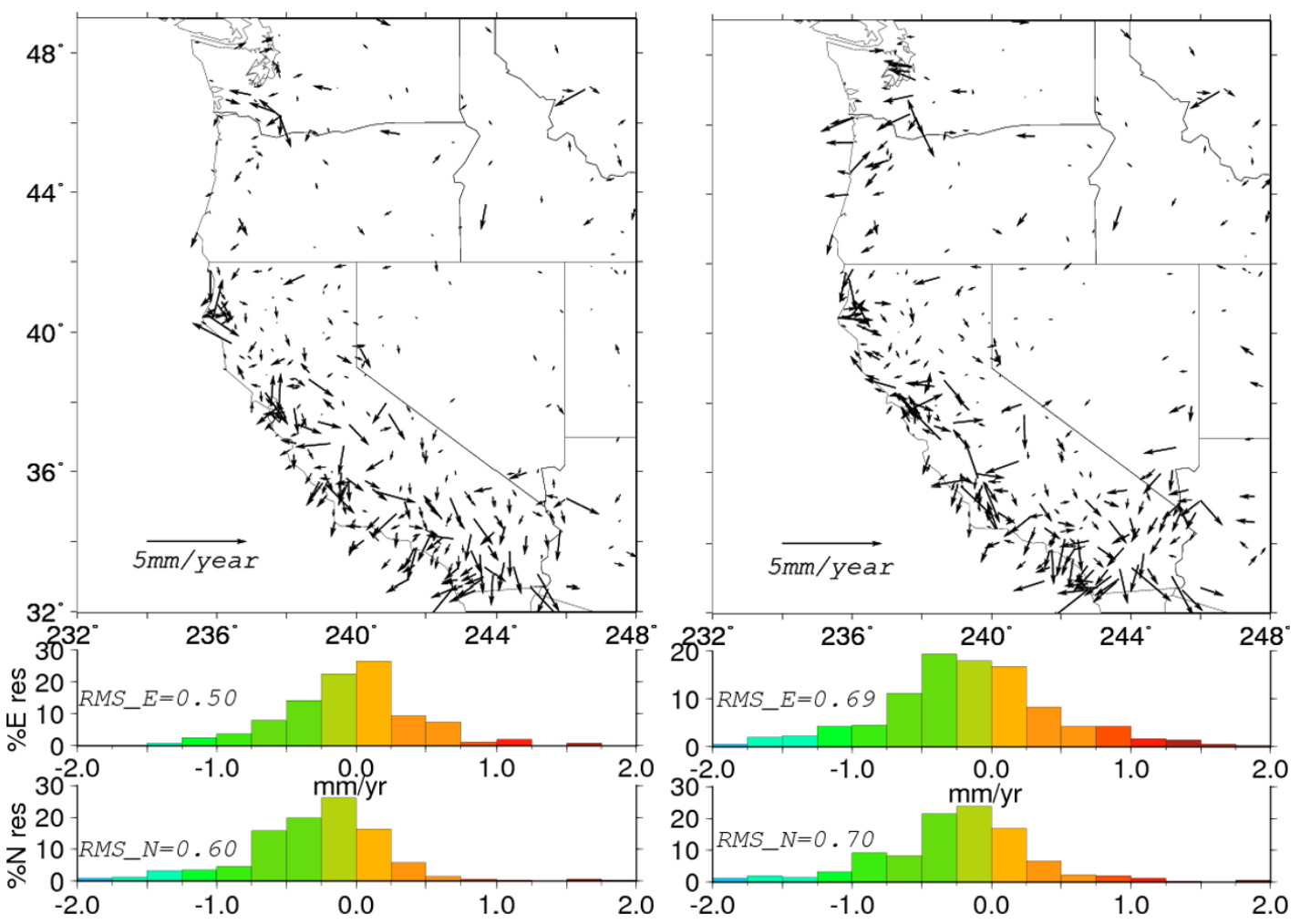

Figure 5: (A: Left) Horizontal velocity residuals with respect to TRANS4D v0.2 and (B: Right) Horizontal velocity residuals with respect to GSRM v2.1. The histograms describe the scatter of the East and North components of the velocity residuals.


Figure 6: (A: Left) Horizontal velocity residuals with respect to TRANS4D v0.2 and (B: Right) Horizontal velocity residuals with respect to GSRM v2.1 in Alaska. The histograms describe the scatter of the East and North components of the velocity residuals.

The residuals $\rho_{t}$ are time-correlated and contain systematic errors. The variances of $P_{0}$ and $v$ were derived assuming colored (general power-law) noise. The modified residuals are computed by adding to the residuals Gaussian white noise with variance equal to that of $P_{0}$ and adding additional Gaussian white noise with variance equal to that of the velocity of the corresponding solution multiplied by $\left(t-t_{0}\right)^{2}$.

The long-term average of the RMS of the modified residuals, i. e. the RMS of the modified residuals divided 


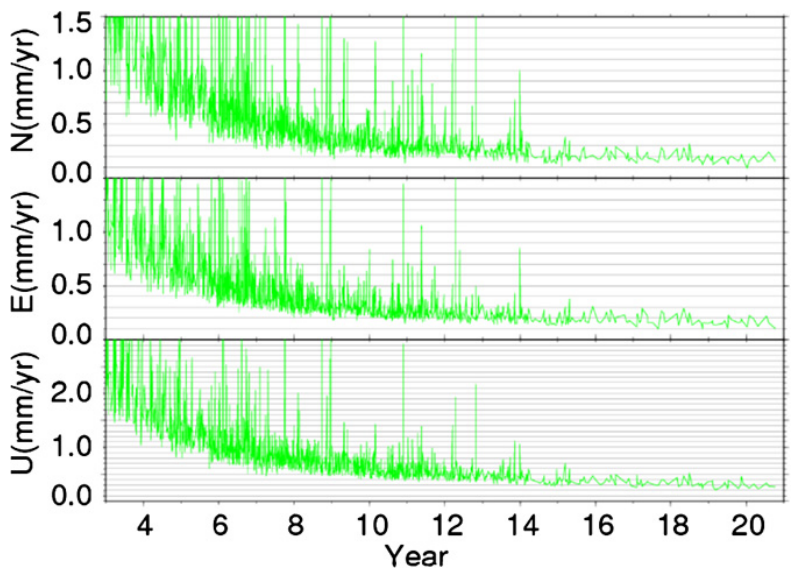

Figure 7: RMS of the modified position residuals per station lifetime in years as a measure of long-term complexity/stability of all operational CORS serving longer than 3 years. Spikes represent stations that are less stable than others of the same lifespan.

by the number of years of operation of the station, is the simplest measure of long-term stability of a station (Fig. 7). Spikes in Fig. 7 represent sites that are less stable than others of similar lifespan. Examination of the time series of the stations causing the spikes exposed 60 stations of the lowest stability (Table S3). After studying the locations and time series of these stations, we could classify them into 9 different categories: (1) Very close to major earthquakes (e.g., MEXI, VERA, ASPA), (2) Affected by manmade non-uniform subsidence (e. g., MMX1, P565, P307, PLSB), (3) Having malfunctioning antenna/equipment (e.g., HRST, MNEY, NELY, NETP), (4) Having significant horizontal instability, likely due to bad monumentation (e. g., TXAN, AB36), (5) Having excessive seasonal behavior (e. g., KYGB, MEWA, PMB1), (6) Located on active volcanoes (e.g., P697, P693), (7) Having nonlinearities caused by strain (e. g., ORES), (8) Having other kinds of nonlinearities, likely hardware-related (e.g., TXBX, MCHN) and (9) Everything else, which includes stations with short lifespan and/or long data gaps and/or slight nonlinearities and/or missed small outliers and/or discontinuities. Section S4 and Figures S10 to S17 present examples of some of the most egregious long-term instabilities. Other complexity measures of the modified residuals, such as differential entropy [35] (see supplementary material section S5), which is based on the entire probability distribution function of the modified residuals (computed using GMT [36]) rather than only its RMS, lead to similar conclusions exposing the very same weak stations (Fig. S18).

For an average CORS (with a lifespan of about 9 years, see Fig. 1), the horizontal stability measures shown in Fig. 7 ( $\sim 0.3$ to $0.4 \mathrm{~mm} /$ year) are only very slightly larger than those derived from kinematics in the previous section (see, e. g., Fig. S8). If this relation extends also to the vertical component of the MYCS2 solution, it would mean that its vertical stability is better than $\sim 0.6 \mathrm{~mm}$ /year.

\section{Summary}

The most recent reprocessing of all daily GPS data collected by the CORS network since 1996, MYCS2, is discussed and used to assess the stability of its stations. This work was done for the purpose of the accurate prediction of station positions to be used for an updated realization of the US NSRS, aligned to IGS14. All well-known phenomena affecting the data (except the second order ionospheric effect) are modeled in MYCS2 while a great effort is made to avoid adverse effects due to poorly understood and hence unmodeled phenomena. We iteratively linearize every nonlinearity, detect every single discontinuity (first automatically but in the last iterations manually), delete every single visible outlier, account for every single spatial correlation between positions, account for colored noise based on reasonable assumptions (general power-law model), and attempt in different ways to minimize aliasing effects due to periodic surface loading.

As a regional reference frame aligned to IGS14, MYCS2 inherits its stability from IGS14. However, additional mismodeling and imperfect assumptions (see, e.g., Fig. S5 and Figs. S9-S17) in the combination and alignment of the MYCS2 to IGS14 can potentially add instability. We estimate the stability of MYCS2 to be better than $0.3 \mathrm{~mm} /$ year in each of the horizontal components and better than $0.6 \mathrm{~mm} /$ year vertically. We show that the combination of horizontal instability and inaccuracy of modern horizontal deformation modeling (at least by both the TRANS4D v0.2 and GSRM v2.1 models) in California is $\sim 0.5-0.7 \mathrm{~mm} /$ year and in Alaska is $\sim 1.4-2.2 \mathrm{~mm} /$ year. For a frame that is realized every $\sim 10$ years, these stability estimates are more than sufficient for mapping and monitoring crustal motion faster than a $\sim 1 \mathrm{~mm} /$ year.

In the supplemental information we show that, excluding stations in very active dynamic regions, our velocity field is consistent with those of 4 other solutions $[37,38,39]$. We then use the results of the reprocessing to expose the poorest stations in the network, including those having malfunctioning equipment. We list the CORS of the poorest stability and present some examples of their poor performance. 
Acknowledgment: The GPS/Orbits infrastructure our work relies upon has been built at NGS and continuously refined since the mid-1980s by Mark Schenewerk, William Dillinger, Gerald Mader, Robert Dulaney, Jim Ray, Stephen Hilla, William Kass, Jake Griffiths, Jim Rhode, Robert (Bud) Hansen, Charlie Schwarz, Clyde Goad and Ben Remondi. The NOAA CORS network has been managed and maintained by William Strange, Neil Weston, Miranda Chin, Mike Cline and Giovanni Sella. We thank colleagues who answered our questions over the years and those who reviewed this paper.

\section{Abbreviations}

$\begin{array}{ll}\text { NA } & \text { North America tectonic plate } \\ \text { US } & \text { United States } \\ \text { NOAA } & \text { Conterminous US } \\ & \text { National Oceanic and Atmospheric Ad- } \\ \text { ministration (US) } \\ \text { NGS } & \text { National Geodetic Survey (US) } \\ \text { GPS } & \text { Global Positioning System } \\ \text { GNSS } & \text { Global Navigation Satellite System } \\ \text { CORS } & \text { Continuously Operating Reference Sta- } \\ & \text { tion }\end{array}$

NSRS

IGS

IGS14

IERS

ITRF2014

IGN

CATREF

SINEX

TEQC

GSRM

GIA

TRANS4D

CATS

RMS

UNR

PBO
MEA, MEaSUREs Making Earth System data records for Use in Research Environments

GMT Generic Mapping Tools

\section{References}

[1] Stone W (2006) The evolution of the National Geodetic Survey's Continuously Operating Reference Station network and Online Positioning User Service, in: Proceedings of IEEE/ION PLANS 2006, San Diego, CA, pp. 653-663.

[2] Strange W, Weston N (1995) The Establishment of a GPS Continuously Operating Reference Station System as a Framework for the National Spatial Reference System, in: Proceedings of the 1995 National Technical Meeting of The Institute of Navigation, Anaheim, CA, January 1995, pp. 19-24.

[3] Griffiths J, Rohde JR, Cline M, Dulaney RL, Hilla S, Kass WG, Ray J, Sella G, Snay R, Soler T (2010) Reanalysis of GPS data for a large and dense regional network tied to a global reference frame, in: IAG Commission 1 Symposium 2010, Reference Frames for Applications in Geosciences (REFAG2010), Marne-La-Vallee, France, October 4-8.

[4] Rebischung P, Schmid R (2016) IGS14/igs14.atx: a new framework for the IGS products, in: AGU Fall Meeting, San Francisco, CA.

[5] NGS (2018) Guidelines for Establishing and Operating CORS. https://www.ngs.noaa.gov/CORS/Establish_Operate_CORS. shtml. NOAA, Silver Spring, MD.

[6] Agnew D, Bock Y, Wyatt F (2001) Comments on NGS Proposed CORS Monumentation, Institute of Geophysics and Planetary Physics, Scripps Institution of Oceanography, University of California, San Diego. https://pfostrain.ucsd.edu/gpsmon/ monument_design/ngscomments.pdf.

[7] Estey LH, Meertens CM (1999) TEQC: the multi-purpose toolkit for GPS/GLONASS data. GPS Solut 3(1):42-49. DOI:10.1007/PL00012778.

[8] Kass W, Dulaney R, Griffiths J, Hilla S, Ray J, Rohde J (2009) Global GPS data analysis at the National Geodetic Survey. J Geod 83:289-295. DOI:10.1007/s00190-008-0255-4.

[9] Schenewerk MS (1991) GPS orbit determination at the National Geodetic Survey, in: Proc. Twenty-Third Precise Time and Time Interval Applications and Planning Meeting, NASA conference publication, vol. 3159, pp. 49-58.

[10] Petit G, Luzum B (2010) IERS Conventions 2010. IERS Tech Note 36, Verlag des Bundesamtes für Kartographie und Geodäsie, Frankfurt am Main, Germany, 179 pp.

[11] Altamimi Z, Sillard P, Boucher C (2012) CATREF software, combination and analysis of terrestrial reference frames, in: IGN, Paris, France.

[12] Blake A, Zisserman A (1987) Visual Reconstruction, MIT Press, Cambridge, MA.

[13] Saleh J (1996) The weak elastic string and some applications in geodesy. J. Geod 70:203-213. DOI:10.1007/BF00873701.

[14] Williams SDP (2003a) Offsets in Global Positioning System time series. J Geophys Res 108(B6):2310. DOI:10.1029/2002JB002156.

[15] Gazeaux J, Williams S, King M, Bos M, Dach R, Deo M, Moore AW, Ostini L, Petrie E, Rogerro M, Teferle FN, Olivares G, Webb 
FH (2013) Detecting offsets in GPS time series: First results from the detection of offsets in GPS experiment. J Geophys Res 118. DOI:10.1002/jgrb.50152.

[16] Altamimi Z, Rebischung P, Métivier L, Collilieux X (2016) ITRF2014: A new release of the International Terrestrial Reference Frame modeling nonlinear station motions. J Geophys Res 121(8):6109-6131. DOI:10.1002/2016JB013098.

[17] van Dam T, Wahr J, Milly PCD, Shmakin AB, Blewitt G, Lavallée D, Larson KM (2001) Crustal displacements due to continental water loading. Geophys Res Lett 28:651-654. DOI:10.1029/2000GL012120.

[18] Dong D, Fang P, Bock Y, Cheng MK, Miyazaki S (2002) Anatomy of apparent seasonal variations from GPS-derived site position time series. J Geophys Res 107(B4), 2075. DOI:10.1029/2001JB000573.

[19] Ray J, Altamimi Z, Collilieux X, van Dam T (2008) Anomalous harmonics in the spectra of GPS position estimates. GPS Solut 12(1):55-64. DOI:10.1007/s10291-007-0067-7.

[20] Collilieux X, Van Dam T, Ray J, Coulot D, Métivier L, Altamimi Z (2012) Strategies to mitigate aliasing of loading signals while estimating GPS frame parameters. J Geodesy 86:1-14. DOI:10.1007/s00190-011-0487-6.

[21] Blewitt G, Lavallée D (2002) Effect of annual signals on geodetic velocity. J Geophys Res 107(B7):ETG 9-1-ETG 9-11. DOI:10.1029/2001JB000570.

[22] Snay RA, Freymueller JT, Craymer MR, Pearson CF, Saleh $J$ (2016) Modelling 3-D crustal velocities in the United States and Canada. J Geophys Res 121:5365-5388. DOI:10.1002/2016JB012884.

[23] Snay RA, Saleh J, Pearson CF (2018) Improving TRANS4D's model for vertical crustal velocities in Western CONUS. J Appl Geod, 12(3):209-227. DOI:10.1515/jag-2018-0010.

[24] Agnew D (1992) The time-domain behavior of power-law noises. Geophys Res Lett 19:333-336. DOI: 10.1029/91GL02832.

[25] Zhang J, Bock Y, Johnson H, Fang P, Williams S, Genrich J, Wdowinski S, Behr J (1997) Southern California permanent GPS geodetic array: Error analysis of daily position estimates and site velocities. J Geophys Res 102(B8):18035-18055. DOI:10.1029/97JB01380.

[26] Mao A, Harrison GA, Dixon TH (1999) Noise in GPS coordinate time series. J Geophys Res 104(B2):2797-2816. DOI:10.1029/1998JB900033.

[27] Williams SDP (2003b) The effect of coloured noise on the uncertainties of rates estimated from geodetic time series. J Geod 76:483-494. DOI:10.1007/s00190-002-0283-4.

[28] Williams SDP, Bock Y, Fang P, Jamason P, Nikolaidis R, Prawirodirdjo L, Miller M, Johnson D (2004) Error analysis of continuous GPS position time series. J Geophys Res 109:B03412. DOI:10.1029/2003JB002741.

[29] Santamaria-Gómez A, Bouin MN, Collilieux X, Wöppelmann G (2011) Correlated errors in GPS position time series: Implications for velocity estimates. J Geophys Res 116:B01405. DOI:10.1029/2010JB007701.
[30] Williams SDP (2008) CATS: GPS coordinate time series analysis software. GPS Solut 12:147-153. DOI:10.1007/s10291-007-0086-4.

[31] Kreemer C, Hammond WC, Blewitt G (2018) A robust estimation of the 3-D intraplate deformation of the north American plate from GPS. J Geophys Res 123:4388-4412. DOI:10.1029/2017JB015257.

[32] Kaufman L, Rousseeuw P (2005) Finding groups in data: An introduction to cluster analysis. John Wiley and Sons, New Jersey.

[33] Kreemer C, Blewitt G, Klein E (2014) A geodetic plate motion and Global Strain Plate Model. Geochem Geophys Geosyst 15:3849-3889. DOI:10.1002/2014GC005407.

[34] Blewitt G, Kreemer C, Hammond WC, Goldfarb JM (2014) Terrestrial reference frame NA12 for crustal deformation studies in North America, J Geodyn 72:11-24. DOI:10.1016/j.jog.2013.08.004.

[35] Cover TM, Thomas JA (2006) Elements of information theory, 2nd edition, John Wiley and Sons, New York.

[36] Wessel P, Smith WHF (1998) New improved version of generic mapping tools released, Eos Trans. AGU, 79(47):579. DOI:10.1029/98E000426.

[37] Blewitt G, Hammond WC, Kreemer C (2018) Harnessing the GPS data explosion for interdisciplinary science. Eos, 99. DOI:10.1029/2018E0104623.

[38] Herring TA, Melbourne TI, Murray MH, Floyd MA, Szeliga WM, King RW, Phillips DA, Puskas CM, Santillan M, Wang L (2016) Plate Boundary Observatory and related networks: GPS data analysis methods and geodetic products. Rev Geophys 54:759-808. DOI:10.1002/2016RG000529.

[39] Bock Y, Webb F (2012) MEaSUREs Solid Earth Science ESDR System, La Jolla, California and Pasadena, California USA. [Available at http://geoapp03.ucsd.edu/gridsphere/ gridsphere, last accessed January 29, 2016].

Supplemental Material: The online version of this article offers supplementary material (https://doi.org/10.1515/jag-2020-0041). The supplementary material includes five sections, eighteen figures and three tables. Section S1 describes the automated edge detection algorithm we used. Section S2 describes the comparison of MYCS2 velocities to other solutions. Section S3 describes the fuzzy clustering method we used to separate spatially stable from unstable CORS. Section S4 presents some examples of long-term unstable CORS, inadequate for mapping control. Finally, section S5 describes differential entropy as a complexity, i.e. instability, measure of position residual time series, which is hopefully more sensitive than the RMS to all stochastic properties of the time series, including time-correlated noise. 